A Martingale Decomposition of Discrete Markov Chains

\author{
Peter Reinhard Hansen
}

CREATES Research Paper 2015-18 


\title{
A Martingale Decomposition of Discrete Markov Chains
}

\section{Peter Reinhard Hansen*}

\author{
European University Institute $\&$ CREATES $S^{\dagger}$
}

April 1, 2015

\begin{abstract}
We consider a multivariate time series whose increments are given from a homogeneous Markov chain. We show that the martingale component of this process can be extracted by a filtering method and establish the corresponding martingale decomposition in closed-form. This representation is useful for the analysis of time series that are confined to a grid, such as financial high frequency data.
\end{abstract}

Keywords: Markov Chain; Martingale; Beveridge-Nelson Decomposition.

JEL Classification: C10; C22; C58

*Address: Department of Economics, European University Institute, Villa San Paolo, Via Della Piazzuola 43,50133 FI Fiesole, Italy. Email: peter.hansen@eui.eu

${ }^{\dagger}$ The author wishes to thank Juan Dolado, James D. Hamilton, an anonymous referee, and seminar participants at Duke University for valuable comments. The author acknowledges support from CREATES - Center for Research in Econometric Analysis of Time Series (DNRF78), funded by the Danish National Research Foundation. 


\section{Introduction}

We consider a $d$-dimensional time series, $\left\{X_{t}\right\}$, whose increments, $\Delta X_{t}=X_{t}-X_{t-1}$, follow a homogeneous ergodic Markov chain with a countable state space. Thus, $X_{t}=X_{0}+\sum_{j=1}^{t} \Delta X_{j}$, which makes $X_{t}$ a (possibly non-stationary) Markov chain on a countable state space. We consider, $\mathrm{E}\left(X_{t+h} \mid \mathcal{F}_{t}\right)$, where $\mathcal{F}_{t}=\sigma\left(X_{t}, X_{t-1}, \ldots\right)$, is the natural filtration. The limit, as $h \rightarrow \infty$, is particularly interesting, because it leads to a martingale decomposition,

$$
X_{t}=Y_{t}+\mu_{t}+U_{t}
$$

where $\mu_{t}$ is a linear deterministic trend, $\left\{Y_{t}, \mathcal{F}_{t}\right\}$ is a martingale with $Y_{t}=\lim _{h \rightarrow \infty} \mathrm{E}\left(X_{t+h}-\mu_{t+h} \mid \mathcal{F}_{t}\right)$, and $U_{t}$ is a bounded stationary process. We derive closed-form expressions for all terms in the representation of $X_{t}$.

The martingale decomposition of finite Markov chains is akin to the Beveridge-Nelson decomposition for ARIMA processes, see Beveridge and Nelson (1981), ${ }^{1}$ and the Granger representation for vector autoregressive processes, see Johansen (1991). The decomposition has many applications, as the longrun properties of $X_{t}$ are governed by the persistent component, $Y_{t}$, while $U_{t}$ characterizes the transitory component of $X_{t}$. In macro-econometrics $Y_{t}$ and $U_{t}$ are often called "trend" and "cycle", respectively, with $Y_{t}$ being interpreted as the long run growth while $U_{t}$ defines the fluctuations around the growth path, see, e.g. Low and Anderson (2008). A martingale decomposition of a stochastic discount process can be used to disentangle economic components with long term and short run impact on asset valuation, see Hansen (2012). For the broader concept of signal extraction of the "trend", see Harvey and Koopman (2002).

In the context with high-frequency financial data (which often are confined to a grid), $Y_{t}$ and $U_{t}$ may be labelled the efficient price and market microstructure noise, respectively. One could use the decomposition to estimate the quadratic variation of the latent efficient price $Y_{t}$, as in Large (2011) and Hansen and Horel (2009), and the framework could be adapted to study market information share, see e.g. Hasbrouck (1995). Markov processes are often used to approximate autoregressive processes in dynamic optimization problems, see Tauchen (1986) and Adda and Cooper (2000), and the decomposition could be used to compare the long-run properties of the approximating Markov process with those of the autoregressive process.

\footnotetext{
${ }^{1}$ The result, known as Beveridge-Nelson decomposition, appeared earlier in the statistics literature, e.g. Fuller (1976, theorem 8.5.1). See Phillips and Solo (1992) for further discussion. The martingale decomposition is also key for the central limit theorem for stationary processes by Gordin (1969).
} 
The paper is organized as follows: We establish an expression for the filtered process within the Markov chain framework, in Section 2, which leads to the martingale decomposition. Concluding remarks with discussion of various extensions are given in Section 3, and all proofs are given in the Appendix.

\section{Theoretical Framework}

In this Section we show how the observed process, $X_{0}, X_{1}, \ldots, X_{n}$, can be filtered in a Markov chain framework, using the natural filtration $\mathcal{F}_{t}=\sigma\left(X_{t}, X_{t-1}, \ldots\right)$. This leads to a martingale decomposition for $X_{t}$ that is useful for a number of things.

Initially we seek the filtered price, $\mathrm{E}\left(X_{t+h} \mid \mathcal{F}_{t}\right)$, and we use the limit, as $h \rightarrow \infty$, to define the process,

$$
Y_{t}=\lim _{h \rightarrow \infty} \mathrm{E}\left(X_{t+h}-\mu_{t+h} \mid \mathcal{F}_{t}\right)
$$

where $\mu_{t}=t \mu$ with $\mu=\mathrm{E}\left(\Delta X_{t}\right)$. We will show that $\left\{Y_{t}, \mathcal{F}_{t}\right\}$ is a martingale, in fact, $Y_{t}$ is the martingale component of $X_{t}$ that, in turn, reveals a martingale representation theorem for finite Markov processes.

Note that the one step increments of $\mathrm{E}\left(X_{t+h}-\mu_{t+h} \mid \mathcal{F}_{t}\right)$ are, in general, autocorrelated at all order (including those lower than $h$ ), however all autocorrelations vanish as $h \rightarrow \infty$ and the martingale property of $Y$ emerges. This filtering argument can be applied to any I(1) process for which $\mathrm{E}\left(\Delta X_{t+h} \mid \mathcal{F}_{t}\right) \stackrel{\text { a.s. }}{\rightarrow} \mathrm{E}\left(\Delta X_{t}\right)$ as $h \rightarrow \infty$, and this is the basic principle that Beveridge and Nelson (1981) used to extract the (stochastic) trend component of ARIMA processes.

\subsection{Notation and Assumptions}

In this section we review the Markov terminology and present our notation that largely follows that in Brémaud (1999, chapter 6). The following assumption is the only assumption we need to make.

Assumption 1. The increments $\left\{\Delta X_{t}\right\}_{t=1}^{n}$ are ergodic and distributed as a homogeneous Markov chain of order $k<\infty$, with $S<\infty$ states.

The assumption that $S$ is finite can be dispensed with, which we detail in Section 3. For now we will assume $S$ to be finite because it greatly simplifies the exposition. The transition matrix for price increments is denoted by $P$. For a Markov chain of order $k$ with $S$ basic states, $P$ will be an $S^{k} \times S^{k}$ matrix. We use $\pi \in \mathbb{R}^{S^{k}}$ to denote the stationary distribution associated with $P$, which is uniquely 
defined by $\pi^{\prime} P=\pi^{\prime}$. The fundamental matrix is defined by ${ }^{2}$

$$
Z=(I-P+\Pi)^{-1}
$$

where $\Pi=\iota \pi^{\prime}$ is a square matrix and $\iota=(1, \ldots, 1)^{\prime}$, (so all rows of $\Pi$ are simply $\pi^{\prime}$ ). We use $e_{r}$ to denote the $r$-th unit vector, so that $e_{r}^{\prime} A$ is the $r$-th row of a matrix $A$ of proper dimensions.

Let $\left\{x_{1}, \ldots, x_{S}\right\}$ be the support for $\Delta X_{t}$, with $x_{s} \in \mathbb{R}^{d}$. We will index the possible realizations for the $k$-tuple, $\Delta \mathcal{X}_{t}=\left(\Delta X_{t-k+1}, \ldots, \Delta X_{t}\right)$, by $\mathbf{x}_{s}, s=1, \ldots, S^{k}$, which includes all the perturbations, $\left(x_{i_{1}}, \ldots, x_{i_{k}}\right), i_{1}, \ldots, i_{k}=1, \ldots, S$. The transition matrix, $P$, is given by

$$
P_{r, s}=\operatorname{Pr}\left(\Delta \mathcal{X}_{t+1}=\mathbf{x}_{s} \mid \Delta \mathcal{X}_{t}=\mathbf{x}_{r}\right)
$$

This matrix will be sparse when $k>1$, because at most $S$ transitions from any state have non-zero probability, regardless of the order of the Markov chain.

For notational reasons it is convenient to introduce the sequence $\left\{s_{t}\right\}$ that is defined by $\Delta \mathcal{X}_{t}=\mathbf{x}_{s_{t}}$, so that $s_{t}$ denotes the observed state at time $t$. We also define the matrix $f \in \mathbb{R}^{S^{k} \times d}$ whose $s$-th row, denoted $f_{s}=e_{s}^{\prime} f$, is the realization of $\Delta X^{\prime}$ in state $s$. It follows that $\Delta X_{t}=f^{\prime} e_{s_{t}}$ and that the expected value of the increments is given by $\mu=\mathrm{E}\left(\Delta X_{t}\right)=f^{\prime} \pi \in \mathbb{R}^{d}$.

The auxiliary vector process, $e_{s_{t}}$, is such that $\mathrm{E}\left(e_{s_{t+1}} \mid \mathcal{F}_{t}\right)=P^{\prime} e_{s_{t}}$, so that $e_{s_{t}}$ can be expressed as a vector autoregressive process of order one with martingale difference innovations, see e.g. Hamilton (1994, p. 679).

\subsection{Markov Chain Filtering}

The filtered process $\mathrm{E}\left(X_{t+h} \mid \mathcal{F}_{t}\right)$, is simple to compute in the Markov setting, because $\mathrm{E}\left(X_{t+h} \mid \mathcal{F}_{t}\right)=$

$\mathrm{E}\left(X_{t+h} \mid \Delta \mathcal{X}_{t}\right)$ and $X_{t+h}=X_{t}+\sum_{j=1}^{h} \Delta X_{t+j}$ with $\mathrm{E}\left(\Delta X_{t+1}^{\prime} \mid \Delta \mathcal{X}_{t}=\mathbf{x}_{r}\right)=\sum_{s=1}^{S^{k}} P_{r, s} f_{s}=e_{r}^{\prime} P f$. More generally we have $\mathrm{E}\left(\Delta X_{t+h}^{\prime} \mid \Delta \mathcal{X}_{t}\right)=e_{s_{t}}^{\prime} P^{h} f$, which shows that

$$
\mathrm{E}\left(X_{t+h}^{\prime} \mid \Delta \mathcal{X}_{t}\right)=X_{t}^{\prime}+e_{s_{t}}^{\prime} \sum_{j=1}^{h} P^{j} f
$$

After subtracting the deterministic trend, $\mu_{t+h}$, we let $h \rightarrow \infty$ and define

$$
Y_{t}=\lim _{h \rightarrow \infty} \mathrm{E}\left(X_{t+h}-\mu_{t+h} \mid \mathcal{F}_{t}\right)
$$

\footnotetext{
${ }^{2}$ The matrix, $I-P+\Pi$, is invertible since the largest eigenvalue of $P-\Pi$ is less than one under Assumption 1 .
} 
which we label the filtered process of $X_{t}$. The process, $Y_{t}$ is well defined and adapted to the filtration $\mathcal{F}_{t}$. We are now ready to formulate our main result.

Theorem 1. The process and $\left\{Y_{t}, \mathcal{F}_{t}\right\}$ is a martingale with initial value, $Y_{0}=X_{0}+f^{\prime}\left(Z^{\prime}-I\right) e_{s_{0}}$ and its increments are given by $\Delta Y_{t}^{\prime}=e_{s_{t}}^{\prime} Z f-e_{s_{t-1}}^{\prime} P Z f$. Moreover, we have

$$
X_{t}=Y_{t}+\mu_{t}+U_{t}
$$

where $U_{t}^{\prime}=e_{s_{t}}^{\prime}(I-Z) f$ is a bounded, stationary, and ergodic process with mean zero.

All terms of the expression are given in closed-form, analogous to the Granger representation theorem by Hansen (2005).

It can be shown that $\Delta Y_{t}$ is a Markov process with $S^{k+1}$ possible states values. Analogous to $P$ and $f$, let $Q$ and $g$ denote the transition matrix for $\Delta Y_{t}$ and its matrix of state values, respectively. The martingale property dictates that $Q g=0 \in \mathbb{R}^{S^{k+1} \times d}$. Note that $\Delta Y_{t}$ is typically conditionally heterogeneous, as $Q$ is not a matrix of rank one, which would be the structure corresponding to the case where $\Delta Y_{t}$ is independent and identically distributed.

The autocovariance structure of the terms in the martingale decomposition is stated next.

Theorem 2. We have $\operatorname{var}\left(\Delta Y_{t}\right)=f^{\prime} Z^{\prime}\left(\Lambda_{\pi}-P^{\prime} \Lambda_{\pi} P\right) Z f$ where $\Lambda_{\pi}=\operatorname{diag}\left(\pi_{1}, \ldots, \pi_{S^{k}}\right)$ and

$$
\operatorname{cov}\left(U_{t}, U_{t+j}\right)=f^{\prime}(I-Z)^{\prime} \Lambda_{\pi} P^{|j|}(I-Z) f=f^{\prime} Z^{\prime} P^{\prime} \Lambda_{\pi} P\left(P^{|j|}-\Pi\right) Z f
$$

and the cross correlations are

$$
\operatorname{cov}\left(\Delta Y_{t}, U_{t+j}\right)=f^{\prime} Z^{\prime}\left(-\Lambda_{\pi}+P^{\prime} \Lambda_{\pi} P\right) P^{j+1} Z f, \quad \text { for } \quad j \geq 0
$$

and $\operatorname{cov}\left(\Delta Y_{t}, U_{t+j}\right)=0$ for $j<0$.

The Theorem shows that the stationary component, $U_{t}$, is autocorrelated and, in general, correlated with current and past (but not future) increments, $\Delta Y_{t}$, of the martingale. In the context of financial high-frequency data, where $U_{t}$ is labelled market microstructure noise, these features are referred to as serially dependent and endogenous noise, that are common empirical characteristics of high-frequency data, see Hansen and Lunde (2006). Let $\lambda_{2}$ denote the second-largest eigenvalue in absolute value of $P$. Since, $\left\|P^{j}-\Pi\right\|=O\left(\left|\lambda_{2}\right|^{j}\right)$ and $\left|\lambda_{2}\right|<1$ under Assumption 1, it follow that the autocovariances of $U_{t}$ decay to zero at an exponential rate. 
A corollary to Theorem 2 is that the following.

Corollary 1. The variance of the observed increments, $\operatorname{var}\left(\Delta X_{t}\right)=f^{\prime}\left(\Lambda_{\pi}-\pi \pi^{\prime}\right) f$ equals

$$
\begin{aligned}
& \operatorname{var}\left(\Delta Y_{t}\right)+2 \operatorname{var}\left(U_{t}\right)-\operatorname{cov}\left(U_{t-1}, U_{t}\right)-\operatorname{cov}\left(U_{t}, U_{t-1}\right)+\operatorname{cov}\left(\Delta Y_{t}, U_{t}\right)+\operatorname{cov}\left(U_{t}, \Delta Y_{t}\right) \\
= & f^{\prime} Z^{\prime}(I-P)^{\prime} \Lambda_{\pi}(I-P) Z f .
\end{aligned}
$$

\section{Concluding Remarks and Extensions}

The martingale decomposition of $X_{t}$ has several applications, as is the case for the Beveridge-Nelson decomposition for ARIMA processes. In the context of macro time series $Y_{t}$ and $U_{t}$ might be labelled the (stochastic) trend and cycle, respectively. In the context of financial high frequency prices, $Y_{t}$ and $U_{t}$ could be labelled the efficient prices and market microstructure noise, respectively. In that context, both $Y_{t}$ and $U_{t}$ are of separate interest. Moreover, extracting the martingale component, $Y_{t}$, offers a motivation for the Markov chain-based estimator of the quadratic variation as in Hansen and Horel (2009). Their estimator is deduced from the long-run variance of $X_{t}$, that facilitates a central limit theory and readily available standard errors.

To conclude, we will discuss extensions of the martingale decomposition to accommodate the cases with an infinite number of states (countable), jumps, and inhomogeneous processes.

Suppose that the number of state values for $\Delta X_{t}$ is countable infinite. Then the number of Markov states for $\Delta \mathcal{X}_{t}$ is countable infinite, and the Markov process can be characterized by $P_{r, s}, r, s=1,2, \ldots$ The concept of ergodicity is well defined, and entails a unique stationary distribution, $\pi$, that satisfies $\pi_{s}=\sum_{r=1}^{\infty} P_{r, s} \pi_{r}$. With $\left[P^{2}\right]_{r, s}=\sum_{j=1}^{\infty} P_{r, j} P_{j, s}$ and higher moments defined similarly, we can define

$$
Z_{r, s}=I_{r, s}+\lim _{h \rightarrow \infty} \sum_{j=1}^{h}\left(\left[P^{j}\right]_{r, s}-\pi_{s}\right)
$$

that are well defined provided that the Markov chain is ergodic. It can now be verified that the expressions in Theorems 1 and 2 continue to be applicable to this case.

In financial time series the increments, $\Delta X_{t}$, are often concentrated about zero, with occasional large changes that are labelled as jumps, see e.g. Huang and Tauchen (2005) and Li (2013). Because jumps are prevalent in high-frequency financial data, the modeling of these data often entails a jump component. One can adapt the martingale decomposition (1) to include a jump component, $J_{t}$. This requires a procedure for classifying large increments as jumps and one can then proceed by removing 
these jumps, e.g. using methods similar to those proposed in Mancini (2009) or Andersen et al. (2012), and then model the remaining returns by the Markov chain methods, to arrive at

$$
X_{t}=Y_{t}+J_{t}+\mu_{t}+U_{t}
$$

where $J_{t}=J_{t-1}+\Delta X_{t} \delta_{j}, \mu_{t}=\mu_{t-1}+\mu\left(1-\delta_{t}\right), U_{t}^{\prime}=\left(1-\delta_{t}\right) e_{s_{t}}^{\prime}(I-Z) f$, with $\delta_{t}$ being the indicator for the jumps.

The case with an inhomogeneous Markov chain is theoretically straightforward provided that the transition matrix, $P_{r, s}(t)=\operatorname{Pr}\left(\Delta \mathcal{X}_{t}=\mathbf{x}_{s} \mid \Delta \mathcal{X}_{t-1}=\mathbf{x}_{r}\right)$, satisfies the ergodicity conditions for all $t$. From the time-varying transition matrix, $P(t)$, one can deduce the increments $\Delta Y_{t}$ and $\Delta \mu_{t}$, as well as $U_{t}$, that

all depend on $P(t)$. A decomposition arises by piecing the terms together, i.e. $Y_{t}=Y_{0}+\sum_{j=1}^{t} \Delta Y_{t}$, and again $Y_{t}$ can be verified to be a martingale, and similarly for other terms. A challenge to implementing this in practice will be to estimate $P(t)$ with a suitable degree of accuracy. This may be achieved by assuming that $P$ is locally homogeneous (piecewise constant), or by imposing a parsimonious structure for the dynamics of $P(t)$, similar to that in the models by Hausman et al. (1992) and Russell and Engle (2005), that can induce an inhomogeneous Markov chain for high-frequency returns.

\section{References}

Adda, J. and Cooper, R. (2000), 'Balladurette and juppette: A discrete analysis of scrapping subsidies', Journal of Political Economy 108, 778-806.

Andersen, T., Dobrev, D. and Schaumburg, E. (2012), 'Jump-robust volatility estimation using nearest neighbor truncation', Journal of Econometrics 169, 75-93.

Beveridge, S. and Nelson, C. R. (1981), 'A new approach to decompositions of time series into permanent and transitory components with particular attentions to measurement of the 'business cycle", Journal of Monetary Economics 7, 151174.

Brémaud, P. (1999), Markov chains: Gibbs fields, Monte Carlo Simulation, and Queues, Springer.

Fuller, W. A. (1976), Introduction to Statistical Time Series, Wiley, New York.

Gordin, M. I. (1969), 'The central limit theorem for stationary processes', Soviet Mathematics Doklady 10, $1174-1176$.

Hamilton, J. D. (1994), Time Series Analysis, Princeton University Press, Princeton N.J.

Hansen, L. P. (2012), 'Dynamic valuation decomposition within stochastic economies', Econometrica 80, 911-967.

Hansen, P. R. (2005), 'Granger's representation theorem: A closed-form expression for I(1) processes', Econometrics Journal 8, 23-38. 
Hansen, P. R. and Horel, G. (2009), 'Quadratic variation by Markov chains', working paper .

Hansen, P. R. and Lunde, A. (2006), 'Realized variance and market microstructure noise', Journal of Business and Economic Statistics 24, 127-218. The 2005 Invited Address with Comments and Rejoinder.

Harvey, A. C. and Koopman, S. J. (2002), 'Signal extraction and the formulation of unobserved components models', Econometrics Journal 3, 84-107.

Hasbrouck, J. (1995), 'One security, many markets: determining the contributions to price discovery', Journal of Finance 50, 1175-1198.

Hausman, J. A., Lo, A. W. and MacKinlay, A. C. (1992), 'An ordered probit analysis of transaction stock prices', Journal of Financial Economics 31, 319-379.

Huang, X. and Tauchen, G. (2005), 'The relative contribution of jumps to total price variation', Journal of Financial Econometrics 3, 456-499.

Johansen, S. (1991), 'Estimation and hypothesis testing of cointegration vectors in gaussian vector autoregressive models', Econometrica 59, 1551-1580.

Large, J. (2011), 'Estimating quadratic variation when quoted prices change by a constant increment', Journal of Econometrics 160, 2-11.

Li, J. (2013), 'Robust estimation and inference for jumps in noisy high frequency data: a local-to-continuity theory for the pre-averaging method', Econometrica 81, 1673-1693.

Low, C. N. and Anderson, H. M. (2008), Economic applications: The beveridge-nelson decomposition, in R. J. Hyndman, A. B. Koehler, J. K. Ord and R. D. Snyder, eds, 'Forecasting with Exponential Smoothing', Springer, chapter 20, pp. 325-337.

Mancini, C. (2009), 'Non-parametric threshold estimation for models with stochastic diffusion coefficient and jumps', Scandinavian Journal of Statistics 36(2), 270-296.

Phillips, P. C. B. and Solo, V. (1992), 'Asymptotics for linear processes', The Annals of Statistics 20, 971-1001.

Russell, J. R. and Engle, R. F. (2005), 'A discrete-state continuous-time model of financial transactions prices and times: The autoregressive conditional multinomial-autoregressive conditional duration model', Journal of Business ES Economic Statistics 23, 166-180.

Tauchen, G. (1986), 'Finite state Markov-chain approximations to univariate and vector autoregressions', Economics Letters 20, $177-181$. 


\section{Appendix of Proofs}

Lemma A.1. Suppose that Assumption 1 holds.

(i) $(P-\Pi)^{j}=P^{j}-\Pi$,

(ii) $\lim _{h \rightarrow \infty} \sum_{j=1}^{h}(P-\Pi)^{j}=Z-I$, where $Z=(I-P+\Pi)^{-1}$,

(iii) $Z \iota=\iota, \pi^{\prime} Z=\pi^{\prime}$, and $P Z=Z P=Z-I+\Pi$,

(iv) $Z-I=(P-\Pi) Z$.

Parts of Lemma A.1 are well know, for instance parts (i) and (ii) are in Brémaud (1999, chapter 6). For the sake of completeness, we include the (short) proofs of all four parts of the Lemma.

Proof. We prove (i) by induction. The identity is obvious for $j=1$. Now suppose that the identity holds for $j$. Then

$$
(P-\Pi)^{j+1}=(P-\Pi)\left(P^{j}-\Pi\right)=P^{j+1}-\Pi P^{j}+\Pi^{2}-P \Pi=P^{j+1}-\Pi,
$$

where the last identity follows from $\Pi P^{j}=\Pi^{2}=P \Pi=\Pi$.

(ii) Since the chain is ergodic we have $\|P-\Pi\|<1$, so that $P^{h}$ converges to $\Pi$ with $\left\|P^{h}-\Pi\right\|=O\left(\left|\lambda_{2}\right|^{h}\right)$, where $\lambda_{2}$ is the second largest eigenvalue of $P$. It follows that $\sum_{j=1}^{\infty}\left(P^{j}-\Pi\right)=\sum_{j=1}^{\infty}(P-\Pi)^{j}$ is absolutely convergent with $\sum_{j=1}^{\infty}(P-\Pi)^{j}=\sum_{j=0}^{\infty}(P-\Pi)^{j}-I=(I-(P-\Pi))^{-1}-I=Z-I$.

(iii) $P^{j} \iota=\iota$ and $\pi^{\prime} P^{j}=\pi^{\prime}$ for any $j \in \mathbb{N}$; and $\Pi \iota=\iota$ and $\pi^{\prime} \Pi=\pi^{\prime}$, so that have $\left(P^{j}-\Pi\right) \iota=\pi^{\prime}\left(P^{j}-\Pi\right)=$

0 . The first two results follow from $Z=I+\sum_{j=1}^{\infty}\left(P^{j}-\Pi\right)$. Next, $P Z=Z P=P+\sum_{j=1}^{\infty}\left(P^{j+1}-\Pi\right)$ and

$$
P+\sum_{j=1}^{\infty}\left(P^{j+1}-\Pi\right)=P+\sum_{j=0}^{\infty}\left(P^{j+1}-\Pi\right)-P+\Pi=\sum_{j=1}^{\infty}\left(P^{j}-\Pi\right)+\Pi=Z-I+\Pi .
$$

Finally, the last result follows from $(Z-I)=\left(I-Z^{-1}\right) Z=(I-I+P-\Pi) Z=(P-\Pi) Z$.

Proof of Theorem 1. We have $\mathrm{E}\left(\Delta X_{t+h}^{\prime} \mid \Delta \mathcal{X}_{t}=\mathbf{x}_{s_{t}}\right)=e_{s_{t}}^{\prime} P^{h} f$. So with $\Delta \mathcal{X}_{t}=\mathbf{x}_{s_{t}}$ we have

$$
\begin{aligned}
\mathrm{E}\left(X_{t+h}-\mu_{t+h} \mid \mathcal{F}_{t}\right) & =X_{t}-\mu_{t}+\sum_{j=1}^{h} \mathrm{E}\left(\Delta X_{t+j}-\mu \mid \mathcal{F}_{t}\right) \\
& =X_{t}-\mu_{t}+f^{\prime} \sum_{j=1}^{h}\left(P^{j}-\Pi\right)^{\prime} e_{s_{t}},
\end{aligned}
$$

where the last term is such that $e_{s}^{\prime} \sum_{j=1}^{h}\left(P^{j}-\Pi\right) f \rightarrow e_{s}^{\prime}(Z-I) f$ as $h \rightarrow \infty$ by Lemma A.1.ii. Hence,

$$
Y_{t}=\lim _{h \rightarrow \infty} \mathrm{E}\left(X_{t+h}-\mu_{t+h} \mid \mathcal{F}_{t}\right)=X_{t}-\mu_{t}+f^{\prime}(Z-I)^{\prime} e_{s_{t}}
$$


so that $Y_{0}=X_{0}+f^{\prime}(Z-I)^{\prime} e_{s_{0}}$ and the increments are given by

$$
\begin{aligned}
\Delta Y_{t}^{\prime} & =\Delta X_{t}^{\prime}-\mu^{\prime}+e_{s_{t}}^{\prime}(Z-I) f-e_{s_{t-1}}^{\prime}(Z-I) f \\
& =e_{s_{t}}^{\prime} Z f-e_{s_{t-1}}^{\prime}(Z+\Pi-I) f=e_{s_{t}}^{\prime} Z f-e_{s_{t-1}}^{\prime} P Z f
\end{aligned}
$$

where we used Lemma A.1.iii.

This establishes the decomposition, $X_{t}=Y_{t}+\mu_{t}+U_{t}$, where $U_{t}^{\prime}=e_{s t}^{\prime}(I-Z) f$. Since $U_{t}$ is a simple function of $\Delta \mathcal{X}_{t}$ it follows that $U_{t}$ is a stationary, ergodic, and bounded process. That $\mathrm{E}\left(U_{t}\right)=0$ follows from $\mathrm{E}\left(U_{t}^{\prime}\right)=\sum \pi_{s} e_{s}^{\prime}(I-Z) f=\left(\pi^{\prime}-\pi^{\prime} Z\right) f=0$, where we used Lemma A.1.iii.

Moreover, $\left\{Y_{t}, \mathcal{F}_{t}\right\}$ is a martingale, because $Y_{t} \in \mathcal{F}_{t}$ and

$$
\mathrm{E}\left(e_{s}^{\prime} Z f-e_{r}^{\prime} P Z f \mid \Delta \mathcal{X}_{t-1}=\mathbf{x}_{r}\right)=\sum_{s} P_{r, s} e_{s}^{\prime} Z f-e_{r}^{\prime} P Z f=e_{r}^{\prime} P Z f-e_{r}^{\prime} P Z f=0
$$

for any $r=1, \ldots, S^{k}$, where $r$ and $s$ are short for $s_{t-1}$ and $s_{t}$, respectively (defined by $\Delta \mathcal{X}_{t-1}=\mathbf{x}_{r}$ and $\left.\Delta \mathcal{X}_{t}=\mathbf{x}_{s}\right)$.

In the proof of Theorem 2 we use the following identities

$$
\sum_{r, s} \pi_{r} P_{r, s} e_{r} e_{r}^{\prime}=\sum_{r, s} \pi_{r} P_{r, s} e_{s} e_{s}^{\prime}=\Lambda_{\pi}, \quad \text { and } \quad \sum_{r, s} \pi_{r}\left[P^{j}\right]_{r, s} e_{r} e_{s}^{\prime}=\Lambda_{\pi} P^{j}
$$

that are easily verified.

Proof of Theorem 2. For the variance of the martingale increments we have

$$
\begin{aligned}
\mathrm{E}\left(\Delta Y_{t} \Delta Y_{t}^{\prime}\right) & =\mathrm{E}\left[\left(f^{\prime} Z^{\prime} e_{s_{t}}-f^{\prime} Z^{\prime} P^{\prime} e_{s_{t-1}}\right)\left(e_{s t}^{\prime} Z f-e_{s_{t-1}}^{\prime} P Z f\right)\right], \\
& =\sum_{r, s} \pi_{r} P_{r, s} f^{\prime} Z^{\prime}\left(e_{s}-P^{\prime} e_{r}\right)\left(e_{s}^{\prime}-e_{r}^{\prime} P\right) Z f \\
& =\sum_{r, s} \pi_{r} P_{r, s} f^{\prime} Z^{\prime}\left(e_{s} e_{s}^{\prime}-e_{s} e_{r}^{\prime} P-P^{\prime} e_{r} e_{s}^{\prime}+P^{\prime} e_{r} e_{r}^{\prime} P\right) Z f \\
& =f^{\prime} Z^{\prime}\left(\Lambda_{\pi}-P^{\prime} \Lambda_{\pi} P-P^{\prime} \Lambda_{\pi} P+P^{\prime} \Lambda_{\pi} P\right) Z f=f^{\prime} Z^{\prime}\left(\Lambda_{\pi}-P^{\prime} \Lambda_{\pi} P\right) Z f,
\end{aligned}
$$

where we used (A.1) in the second last equality. 
Concerning the stationary component of the decomposition we have for $j \geq 0$ that

$$
\begin{aligned}
\mathrm{E}\left(U_{t} U_{t+j}^{\prime}\right) & =\mathrm{E}\left[f^{\prime}(I-Z)^{\prime} e_{s t} e_{s_{t+j}}^{\prime}(I-Z) f\right] \\
& =\sum_{r, s} \pi_{r}\left[P^{j}\right]_{r, s} f^{\prime}(I-Z)^{\prime} e_{r} e_{s}^{\prime}(I-Z) f \\
& =f^{\prime}(I-Z)^{\prime} \Lambda_{\pi} P^{j}(I-Z) f \\
& =f^{\prime} Z^{\prime}(\Pi-P)^{\prime} \Lambda_{\pi} P^{j}(\Pi-P) Z f \\
& =f^{\prime} Z^{\prime} P^{\prime} \Lambda_{\pi} P\left(P^{j}-\Pi\right) Z f
\end{aligned}
$$

where we used Lemma A.1.iv in the second last equality.

Finally, for the cross covariance we first note that,

$$
\begin{aligned}
& \sum_{r, s, v} \pi_{r} P_{r, s}\left[P^{j}\right]_{s, v} e_{s} e_{v}^{\prime}=\sum_{s, v} \pi_{s}\left[P^{j}\right]_{s, v} e_{s} e_{v}^{\prime}=\Lambda_{\pi} P^{j} \\
& \sum_{r, s, v} \pi_{r} P_{r, s}\left[P^{j}\right]_{s, v} e_{r} e_{v}^{\prime}=\sum_{r, s, v} \pi_{r}\left[P^{j+1}\right]_{r, v} e_{r} e_{v}^{\prime}=\Lambda_{\pi} P^{j+1}
\end{aligned}
$$

where the first identities in the two equations follow by $\sum_{r} \pi_{r} P_{r, s}=\pi_{s}$ and $\sum_{s} P_{r, s}\left[P^{j}\right]_{s, v}=\left[P^{j+1}\right]_{r, v}$, respectively, and the last equalities both follow from the last variant of (A.1). So for $j \geq 0$ we have

$$
\begin{aligned}
\mathrm{E}\left(\Delta Y_{t} U_{t+j}^{\prime}\right) & =\mathrm{E}\left[\left(e_{s_{t}}^{\prime} Z f-e_{s_{t-1}}^{\prime} P Z f\right)^{\prime} e_{s_{t+j}}^{\prime}(I-Z) f\right] \\
& =\sum_{r, s, v} \pi_{r} P_{r, s}\left[P^{j}\right]_{s, v} f^{\prime} Z^{\prime}\left(e_{s}-P^{\prime} e_{r}\right) e_{v}^{\prime}(\Pi-P) Z f \\
& =f^{\prime} Z^{\prime}\left[\Lambda_{\pi} P^{j}(\Pi-P)-P^{\prime} \Lambda_{\pi} P^{j+1}(\Pi-P)\right] Z f \\
& =f^{\prime} Z^{\prime}\left[\pi \pi^{\prime}-\Lambda_{\pi} P^{j+1}-\pi \pi^{\prime}+P^{\prime} \Lambda_{\pi} P^{j+2}\right] Z f \\
& =f^{\prime} Z^{\prime}\left(-\Lambda_{\pi}+P^{\prime} \Lambda_{\pi} P\right) P^{j+1} Z f .
\end{aligned}
$$

That $\mathrm{E}\left(\Delta Y_{t} U_{t+j}^{\prime}\right)=0$ for $j<0$ can be verified similarly. However, this is not required because the zero covariances are a simple consequence of martingale property of $Y_{t}$ that was established in the proof of Theorem 1.

Proof of Corollary (1). By substituting the expressions from Theorem 2 and using $\operatorname{cov}\left(\Delta Y_{t}, U_{t-1}\right)=$ 
0 , one finds that the expression in Corollary 1 equals $f^{\prime} Z^{\prime} A Z f$, where

$$
\begin{aligned}
A= & \left(\Lambda_{\pi}-P^{\prime} \Lambda_{\pi} P\right)+2 P^{\prime} \Lambda_{\pi} P(I-\Pi)-P^{\prime} \Lambda_{\pi} P(P-\Pi)-(P-\Pi)^{\prime} P^{\prime} \Lambda_{\pi} P \\
& +\left(-\Lambda_{\pi}+P^{\prime} \Lambda_{\pi} P\right) P+P^{\prime}\left(-\Lambda_{\pi}+P^{\prime} \Lambda_{\pi} P\right) \\
= & \Lambda_{\pi}+P^{\prime} \Lambda_{\pi} P-2 \pi \pi^{\prime}+\pi \pi^{\prime}+\pi \pi^{\prime}-\Lambda_{\pi} P-P^{\prime} \Lambda_{\pi} \\
= & (I-P)^{\prime} \Lambda_{\pi}(I-P)
\end{aligned}
$$

which proves the equality in the Corollary. That $f^{\prime} Z^{\prime} A Z f=f^{\prime}\left(\Lambda_{\pi}-\pi \pi^{\prime}\right) f$ follows from

$$
\begin{aligned}
(I-P)^{\prime} \Lambda_{\pi}(I-P) & =(I-P+\Pi)^{\prime} \Lambda_{\pi}(I-P+\Pi)-\pi \pi^{\prime} \\
& =(I-P+\Pi)^{\prime}\left(\Lambda_{\pi}-\pi \pi^{\prime}\right)(I-P+\Pi),
\end{aligned}
$$

which equals $\left(Z^{-1}\right)^{\prime}\left(\Lambda_{\pi}-\pi \pi^{\prime}\right) Z^{-1}$. This completes the proof. 
2015-01 Tom Engsted, Simon J. Hviid and Thomas Q. Pedersen: Explosive bubbles in house prices? Evidence from the OECD countries

2015-02: Tim Bollerslev, Andrew J. Patton and Wenjing Wang: Daily House Price Indices: Construction, Modeling, and Longer-Run Predictions

2015-03: Christian M. Hafner, Sebastien Laurent and Francesco Violante: Weak diffusion limits of dynamic conditional correlation models

2015-04: $\quad$ Maria Eugenia Sanin, Maria Mansanet-Bataller and Francesco Violante: Understanding volatility dynamics in the EU-ETS market

2015-05: Peter Christoffersen and Xuhui (Nick) Pan: Equity Portfolio Management Using Option Price Information

2015-06: $\quad$ Peter Christoffersen and Xuhui (Nick) Pan: Oil Volatility Risk and Expected Stock Returns

2015-07: Peter Christoffersen, Bruno Feunou and Yoontae Jeon: Option Valuation with Observable Volatility and Jump Dynamics

2015-08: $\quad$ Alfonso Irarrazabal and Juan Carlos Parra-Alvarez: Time-varying disaster risk models: An empirical assessment of the Rietz-Barro hypothesis

2015-09: Daniela Osterrieder, Daniel Ventosa-Santaulària and Eduardo Vera-Valdés: Unbalanced Regressions and the Predictive Equation

2015-10: $\quad$ Laurent Callot, Mehmet Caner, Anders Bredahl Kock and Juan Andres Riquelme: Sharp Threshold Detection Based on Sup-norm Error rates in Highdimensional Models

2015-11: $\quad$ Arianna Agosto, Giuseppe Cavaliere, Dennis Kristensen and Anders Rahbek: Modeling corporate defaults: Poisson autoregressions with exogenous covariates (PARX)

2015-12: $\quad$ Tommaso Proietti, Martyna Marczak and Gianluigi Mazzi: EuroMlnd-D: A Density Estimate of Monthly Gross Domestic Product for the Euro Area

2015-13: $\quad$ Michel van der Wel, Sait R. Ozturk and Dick van Dijk: Dynamic Factor Models for the Volatility Surface

2015-14: Tim Bollerslev, Andrew J. Patton and Rogier Quaedvlieg: Exploiting the Errors: A Simple Approach for Improved Volatility Forecasting

2015-15: Hossein Asgharian, Charlotte Christiansen and Ai Jun Hou: Effects of Macroeconomic Uncertainty upon the Stock and Bond Markets

2015-16: Markku Lanne, Mika Meitz and Pentti Saikkonen: Identification and estimation of non-Gaussian structural vector autoregressions

2015-17: $\quad$ Nicholas M. Kiefer and C. Erik Larson: Counting Processes for Retail Default Modeling

2015-18: $\quad$ Peter Reinhard Hansen: A Martingale Decomposition of Discrete Markov Chains 